\title{
Dynamic scan control in STEM: spiral scans
}

\author{
Xiahan Sang ${ }^{1,2+}$, Andrew R. Lupini ${ }^{2,3^{*}}$, Raymond R. Unocic ${ }^{1,2}$, Miaofang Chi ${ }^{1,2}$, Albina Y. Borisevich ${ }^{2,3}$, \\ Sergei V. Kalinin ${ }^{1,2}$, Eirik Endeve ${ }^{4}$, Richard K. Archibald ${ }^{4}$ and Stephen Jesse ${ }^{1,2^{*}}$
}

\begin{abstract}
Scanning transmission electron microscopy (STEM) has emerged as one of the foremost techniques to analyze materials at atomic resolution. However, two practical difficulties inherent to STEM imaging are: radiation damage imparted by the electron beam, which can potentially damage or otherwise modify the specimen and slow-scan image acquisition, which limits the ability to capture dynamic changes at high temporal resolution. Furthermore, due in part to scan flyback corrections, typical raster scan methods result in an uneven distribution of dose across the scanned area. A method to allow extremely fast scanning with a uniform residence time would enable imaging at low electron doses, ameliorating radiation damage and at the same time permitting image acquisition at higher frame-rates while maintaining atomic resolution. The practical complication is that rastering the STEM probe at higher speeds causes significant image distortions. Non-square scan patterns provide a solution to this dilemma and can be tailored for low dose imaging conditions. Here, we develop a method for imaging with alternative scan patterns and investigate their performance at very high scan speeds. A general analysis for spiral scanning is presented here for the following spiral scan functions: Archimedean, Fermat, and constant linear velocity spirals, which were tested for STEM imaging. The quality of spiral scan STEM images is generally comparable with STEM images from conventional raster scans, and the dose uniformity can be improved.
\end{abstract}

Keywords: Aberration-corrected STEM, Scan control, Distortion, Spiral scan

\section{Background}

Beam damage, drift distortion, and scan distortion are inherent issues that hinder quantitative interpretation of scanning transmission electron microscopy (STEM) imaging [1-6]. Beam damage occurs when the electron beam used to form the image transfers a critical amount of energy to the sample being examined, potentially causing damage or otherwise changing the subject of the experiment. This effect can be very useful, for example, allowing electron energy loss spectroscopy (EELS), or for the deliberate sculpting of nano-device components [7], or to excite diffusion of single atoms $[8,9]$ and vacancies

\footnotetext{
*Correspondence: arl1000@ornl.gov; sjesse@ornl.gov

${ }^{+}$Xiahan Sang and Andrew R. Lupini contributed equally to the paper

1 Center for Nanophase Materials Sciences, Oak Ridge National Laboratory, Oak Ridge, TN 37831, USA

${ }^{3}$ Materials Sciences and Technology, Oak Ridge National Laboratory, Oak Ridge, TN 37831, USA

Full list of author information is available at the end of the article
}

[10]. However, in most cases, such damage to the sample is usually considered to be detrimental. Thus, various strategies are employed to minimize beam damage, and the optimal method will depend on the properties of the sample and microscope imaging parameters. If damage is dominated by knock-on mechanisms, a viable option is to reduce the accelerating voltage below the threshold at which significant damage occurs. Conversely, if the damage is dominated by ionization, then it may be beneficial to increase the accelerating voltage to reduce the ionization cross section [1]. Additional experimental procedures might also be useful, such as coating the sample with a conductive layer (such as carbon), imaging inside a liquid [11], or operating at cryogenic temperature [12].

Similarly, a variety of imaging strategies can be employed to minimize the electron dose, chief among which is reducing the beam current. Using more source demagnification can improve spatial resolution, but the lower signal level may degrade the signal-to-noise ratio. 
Other possibilities include control of the beam dose via 'blanking', adjusting operating parameters (such as focus and astigmatism) on an area slightly away from the area of interest, using repeated fast scans [13], or making more efficient use of the available signals [14]. The recent development of sparse sampling methods also appears to be extremely promising [15].

On the other hand, acquisition of multiple fast scans can both reduce the dose rate and allow sequential imaging, which is particularly useful for samples that are beamsensitive or that experience charging. Also, there has been a recent resurgence of interest in applying methods to correct scan and drift distortions in STEM using frame averaging [2-6]. However, the success of these methods raises the question of whether the scan itself can be improved to eliminate some of the distortions during data acquisition rather than by post-processing. Extremely high-speed scanning and the possibility of dynamic stabilization seem to be promising routes for further exploration.

Advantages of using non-traditional scan paths have been demonstrated in scanning probe microscopy (SPM), including improved speed and accuracy and the ability to automate the targeting of regions of interest for higher resolution measurements [16, 17]. However, customization or optimization of the scanning path has rarely been used in STEM. There are several technical difficulties associated with scanning in STEM. These mostly arise because of the competing demands on the probe response: the user might wish to move the probe rapidly, requiring a fast response, whereas the probe also has to be highly stable and not wobble about each position during a slower scan. Typically, the scan speed used for spectroscopy might be 3-6 orders of magnitude slower than for imaging. Obviously, these competing demands place stringent requirements on the scan amplification electronics. Moreover, STEM scans are usually 'doubledeflection' to obtain tilt-free scans or coma-free scans. Here, we will largely ignore such details and treat the magnification and scan purification as separate problems.

In this paper, we show for the first time that aberrationcorrected STEM images can be formed at high speed using paths that are significantly different from traditional orthogonal rastering. Advantages and disadvantages of different scan paths will be compared in terms of sampling uniformity and distortion. To differentiate from conventional rastering mode scans, this new scanning method will be referred to as general-scan STEM (G-STEM).

\section{Experimental}

For test purposes, we used a $\mathrm{SrTiO}_{3}$ (STO) sample viewed down the [110] zone axis. STO is a very common substrate for thin-film growth, which is a major topic of interest for electron microscopy, meaning that there will likely be an STO reference region available on many technologically important samples. Moreover, STO is reasonably stable and does not charge significantly under typical electron doses.

STEM images were acquired used an FEI Titan 80-300 operating at $300 \mathrm{kV}$ equipped with a Fischione high-angle annular dark-field (HAADF) detector. We developed a custom field-programmable gate array (FPGA)-based scan system (in a National Instruments PXIe-1073 chasis) capable of interfacing to a variety of different microscopes. A LabView program was developed to control the scan unit with input coordinates from customizable Matlab code. This system generates voltage waveforms that are sent to the $\mathrm{x}$ - and $\mathrm{y}$ - scan controls to enable arbitrary and dynamic beam positioning. The maximum readout frequency of the FPGA scan system is $2 \mathrm{MHz}$ with an equivalent shortest dwell time of $0.5 \mu \mathrm{s}$.

\section{Results}

\section{Data representation}

At this stage, it is important to point out that the unconventional scan patterns used here induce a paradigm shift in how image data are considered. In a traditional scanning mode, the data are essentially stored as an array of intensities, which are assigned to elements within a 2D matrix. However, for more complicated scan patterns, it is also necessary to specify the (nominal) position where each data point was acquired. A simple interpolation algorithm (herein called reconstruction) is used to map each data point to an element of the displayed or printed image. Thus, rather than a simple list of intensities $\left(I_{i}\right)$, the data are better envisioned as a list of positions and intensities $\left(x_{i}, y_{i}, I_{i}\right)$.

In practice, we have begun to store the nominal positions in this manner. Of course, it is possible to just store the scan-generation algorithm, but the factor of 3 increases in storage requirements is largely irrelevant here. Moreover, if distortions are significant, the true probe position may be quite different from the nominal position. Scan distortion correction consists of constructing the map from nominal to 'true' probe positions. Thus, this paradigm also highlights the analogy to the usual post-processing distortion correction, where a perpixel map of corrections is generated [2-4].

In this paper, every G-STEM data set contains a series of twenty frames each acquired with $0.2 \mathrm{~s}$ frame time and the maximum frequency of $2 \mathrm{MHz}$. The 400,000 data points in each frame were then reconstructed to form a $200 \times 200$ image. The twenty image frames were aligned using cross-correlation and averaged to increase the signal-to-noise ratio (SNR). The final images presented in the figures were further smoothened in the frequency domain using a Gaussian filter. 


\section{Sawtooth scans}

A typical STEM image acquired using the conventional raster scan path with a dwell time of $20 \mu$ s and $512 \times 512$ frame size is shown in Fig. 1a. Here, the brighter atom columns are $\mathrm{Sr}$ and the fainter columns are Ti. The drift distortion is evident as the angle between [110] and [001] deviates from $90^{\circ}$. We start the G-STEM attempt from the simple sawtooth scan path that resembles conventional raster scan from left to right and top to bottom. Here, we use a simple version of this path such that the beam flies directly from the end of the last line to the start of the next line and
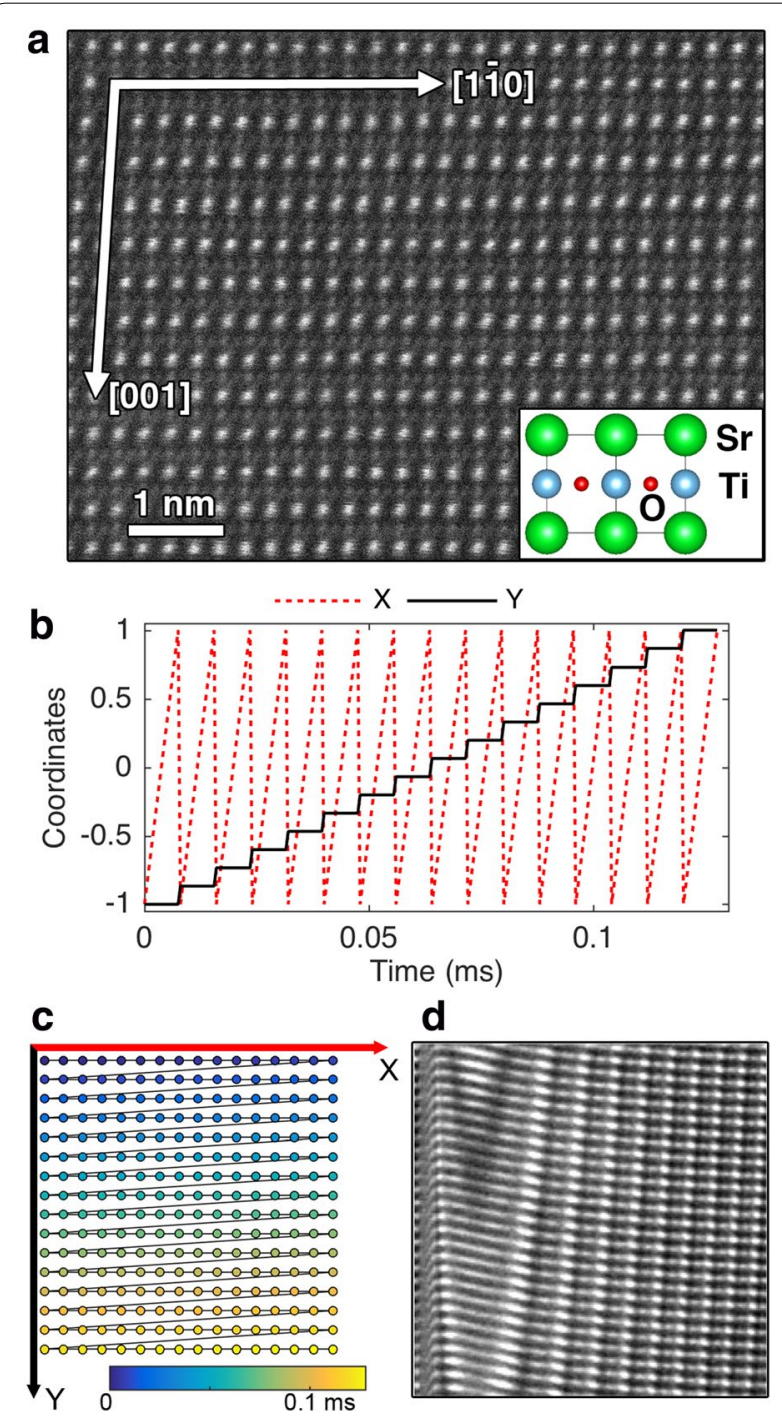

Fig. 1 a Conventional STEM image acquired from STO along [110] zone axis. Schematic illustration of a sawtooth scan. $\mathbf{b}$ Voltages applied to the $\mathbf{X}$ and $\mathbf{Y}$ scan coils over the time for a single frame acquisition. c Probe positions, shaded from dark to light as a function of time. $\mathbf{d}$ A reconstructed G-STEM image acquired with a sawtooth scan continues to scan without any flyback time or line synchronization. The probe location $\left(x_{i}, y_{i}\right)$ as a function of time is shown in Fig. 1b. Here, $\left(x_{i}, y_{i}\right)$ scales to the voltages applied along the two directions. The $\mathbf{X}$-axis (red) is defined as the horizontal direction, also known as the fast scan direction in the conventional STEM. The Y-axis (black) is defined as the vertical direction, also known as the slow scan direction. The scans for both the $\mathbf{X}$ - and $\mathbf{Y}$-axes are sawtooth waves of appropriate frequencies. Practically, the amplitude of this wave is controlled by the microscope electronics and defines the magnification of the STEM image. To better illustrate the scan path, we also plot the beam locations in 2D, as shown in Fig. 1c. The black zigzagging line connecting the dots illustrates the scan path.

Figure 1d shows a processed G-STEM image acquired using a fast sawtooth scan with a frame time of $0.2 \mathrm{~s}$ and 20 frames as discussed earlier. Note that although we use the HAADF signal in this paper, it is possible to simultaneously acquire multiples signals, such as both brightand dark-field signals. The image is significantly distorted at the left edge of the displayed region, although the rest of the image is relatively undistorted. This distortion is likely from the phase lag of the scan electronics responding to a sudden change of beam location. When the beam moves from the end of the last line to the start of the next line, the actual location will take some extra time to reach the nominal position. Therefore, one way to compensate for the lag is to add in some extra shifts or a delay time, as in a conventional cathode ray tube.

Conventionally, a 'flyback' delay at the start of each fast scan line is used to reduce such distortion. For a present state-of-the-art STEM, flyback delays of 10-1000 $\mu$ s are typical. As a specific example, the Nion UltraSTEM 200 typically needs more than $500 \mu$ s to yield images without noticeable distortions. Thus, for a scan of $512 \times 512$ pixels at $1 \mu \mathrm{s} /$ pixel, using this flyback delay would result in losing roughly half of the available imaging time. If a fast enough blanker is available, the beam could be blanked during the flyback; otherwise, there might also be additional unnecessary damage at the edges of the scan where the beam spends extra time. The distribution of the electron dose is an important topic that will recur later. Clearly, a method of eliminating the flyback delay would allow an increase in scanning rate and potentially reduce the beam damage.

Another method to reduce the distortion and lateral shift along slow scan direction is called line-synchronization, i.e., tying each line to the same part of the wave of the electrical supply. Such synchronization has the added advantage that the effects of mains interference should be similar for each scan line and each frame, facilitating its correction [18]. However, this method either requires a delay time at the start of each line or imposes additional restrictions on the per-pixel dwell time. 


\section{Serpentine scans}

An obvious improvement over the sawtooth scan to avoid a flyback delay is to perform a 'serpentine' scan, alternately moving the probe from left to right on one scan line and then right to left on the next, using what is sometimes called a triangle wave. A serpentine scan is shown in Fig. 2a, b, where the $\mathbf{X}$ - and $\mathbf{Y}$ - directions are the same as in Fig. 1b. Double serpentine scans (i.e., performing a second scan after rotating the slow-scan axis by $90^{\circ}$ ) can also be implemented.

Figure 2c shows the result of such a serpentine scan. Unfortunately, these scans initially appear worse than the conventional scan at high scan speeds, because the distortions are different for the leftwards and rightwards trajectories. For display purposes, it is best to separate out these two paths. Notably, unwarping this distortion might present an easier problem to solve than the regular sawtooth wave, because the triangle wave provides two images of the same area with different distortions. To a

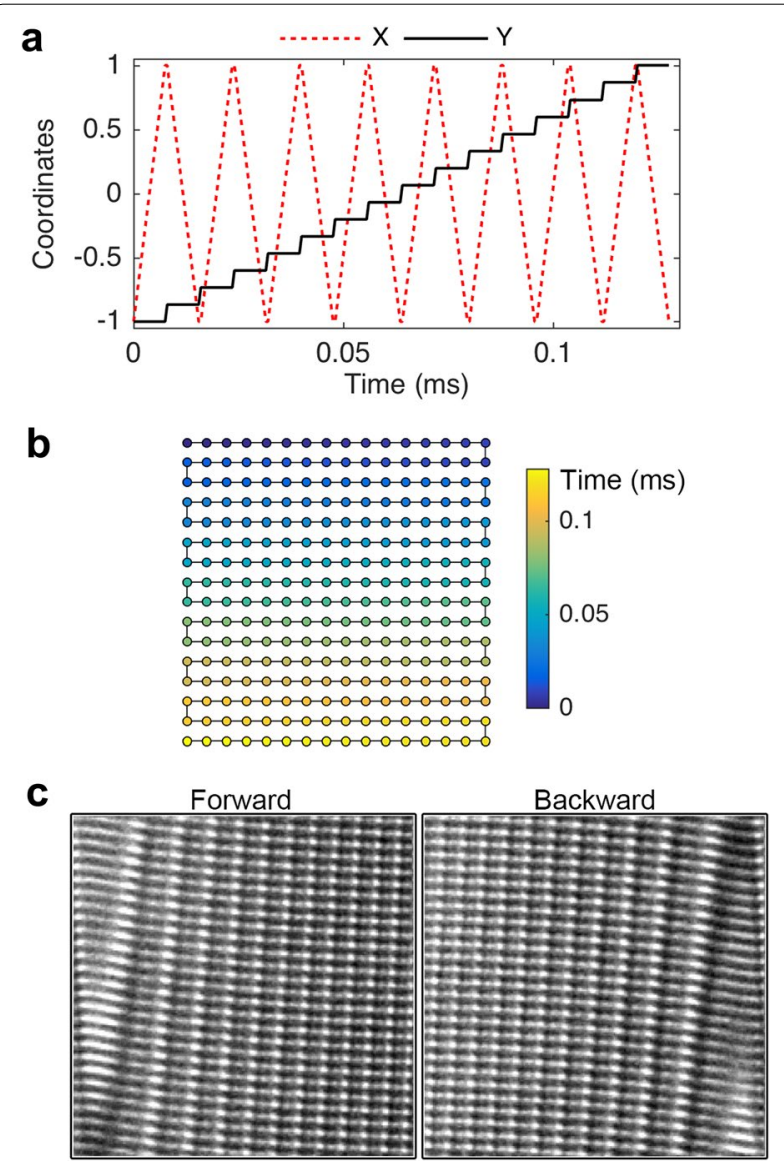

Fig. 2 Schematic illustration of a serpentine scan. a Voltages applied to the $X$ and $Y$ scan coils over the time for a single frame. $\mathbf{b}$ Probe positions, shaded from dark to light as a function of time. c Reconstructed G-STEM images (forward and backward) acquired with a serpentine scan reasonable level of approximation, we might, therefore, expect the distortions to be similar, but reversed. Thus, a digital correction of serpentine scans could be a promising route for further development.

The obvious lesson from the serpentine scans is that the sharp changes in direction at the edges of the scan contribute significantly to the distortions. There is a clear difference in the acceleration of the probe between the abrupt changes at the end of each scan line as compared with the rest of the pixels. The relevance should be obvious in scanning tunneling microscopy (STM), in which the moving probe/stage has mass, but is perhaps a little surprising in STEM where the 'probe' does not really correspond to a physical object. However, it seems clear that there is a non-ideal response of the 'true' probe movement to the 'nominal' probe positions. The cause of this lag is inductance in the scan coils and other current-flow limitations, which limit how fast the scan can be changed, in an analogous way as to how inertia can limit mechanical movement. One route to address this problem would be with faster electronics or rapid electrostatic deflectors. However, such new hardware would introduce other complications and, thus, scan paths without sharp changes in acceleration merit further investigation.

\section{Spiral scans}

We now focus on smooth curves that can fill the 2D space without crossing themselves. The distortions can hopefully be reduced due to the relatively smooth acceleration. Spiral curves are natural solutions to this problem. The mathematical study of spirals has a long and interesting history, dating back thousands of years [19]. In this paper, we focus on spirals with coordinates $(x, y)$ as a function of time $t$ defined by:

$$
x=t^{a} \cos \left(\omega t^{b}\right), y=t^{a} \sin \left(\omega t^{b}\right)
$$

where $\omega$ is the scanning frequency, and $a$ and $b$ are parameters to control the shape of the spirals. The scanning frequency $\omega$ can be adjusted to change the sampling rate. The spiral can go both inward and outward. As the drift distortion is different but correlated for inward and outward scans, this is a promising way to decouple drift distortion from the scan distortion, which will be considered in more detail in the future work.

Here, we explore the physical properties of the spiral curves, as they are closely related to the quality of STEM images reconstructed from those scan paths. We begin with the velocity $\vec{v}$, which basically determines the distance between adjacent sampling points. For each point on the spiral, $\vec{v}$ is the first derivative of Eq. (1), with a magnitude: 


$$
|\vec{v}|=t^{a-1} \sqrt{a^{2}+\omega^{2} b^{2} t^{2 b}} \approx \omega b t^{a+b-1}
$$

The term $a^{2}$ inside the square root can usually be neglected for large $\omega t^{b}$. We can see that when $a+b=$ 1 the velocity magnitude is approximately constant for all the points on the spiral. If $a+b>1$, the beam moves faster as it moves away from the center.

The angular velocity magnitude $\Omega$ is defined by:

$$
\Omega=\frac{|\vec{v}|}{r}=\frac{\omega b t^{a+b-1}}{t^{a}}=\omega b t^{b-1}
$$

Here, we assume that the velocity $\vec{v}$ is perpendicular to $\vec{r}=(x, y)$, which is a reasonable approximation: The angle between $\vec{v}$ and $\vec{r}$ can be calculated as $\theta=\arccos (a)$ $\left.\left(\omega b t^{b}\right)\right)$. As $t$ increases, $\cos (\theta)$ approaches zero and $\theta$ approaches $90^{\circ}$. Equation 3 tells us that the angular velocity is approximately constant if $b=1$.

Another potentially interesting feature of the spiral curves is the sampling density. To ensure uniform sampling, the dose should ideally be the same across the whole area. For a first approximation, we consider how the spiral sweeping area $A$ increases as a function of time $t$,

$$
\frac{d A}{d t}=\frac{d\left(\pi\left(t^{a}\right)^{2}\right)}{d t}=2 a \pi t^{2 a-1}
$$

For $a=0.5$, the area increases linearly with time. For $a<0.5$, the increase slows down over time, resulting in more dose at the edges, while for $a>0.5$, the center is exposed to more electron dose. Now, with the understanding of physical properties of the spiral scans, we investigate the behavior for spiral curves with different $a$ and $b$ parameters.

\section{Archimedean spiral}

The first type of spiral we consider is an 'Archimedean' spiral with $a=1$ and $b=1$ :

$$
x=t \cos (\omega t), y=t \sin (\omega t)
$$

The beam scan path Fig. 3a shows that the magnitude of $x$ and $y$ slowly increase without any sharp turns and the frequency of the sinusoids remains constant. Taking coordinates from Fig. 3a, we can form the outward scan trajectory, as shown in the left part of Fig. 3b. The inward scan shown in the right part is constructed by reversing of the scan path and also the y-direction. Two typical reconstructed images using Archimedean inward and outward spirals are shown in Fig. 3c. Note that the resulting STEM images do not display any obvious non-linear distortion. This is attributed to the constant frequencies (and constant angular velocity) for $b=1$. Both inward and outward images are rotated at the same angle with

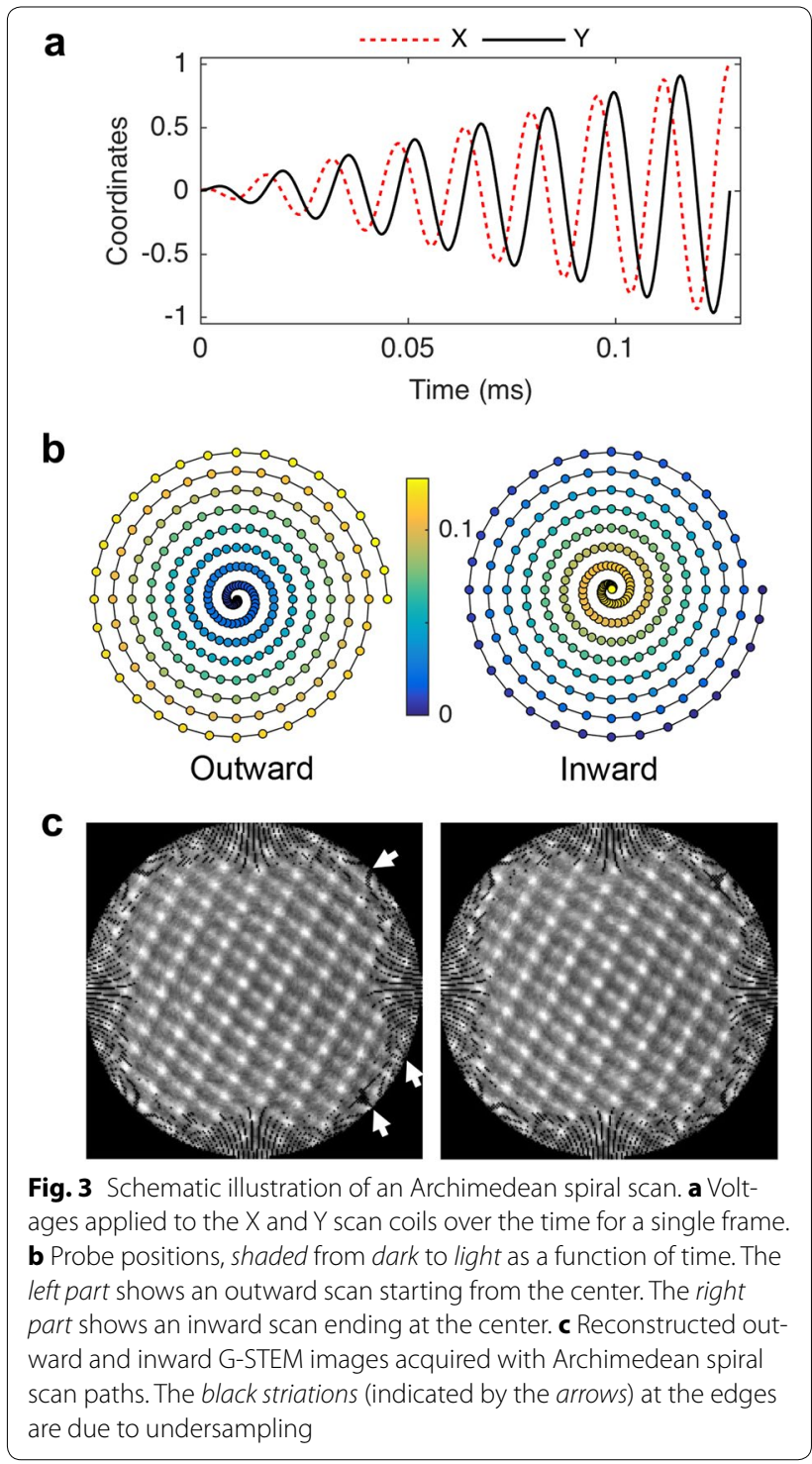

respect to the sawtooth scan images in Fig. 1d. The distortion is likely from the scan lag which is related to the angular velocity. As the spiral scan direction is clockwise for both the inward and outward scans, the distortion is the same for both images.

The main problem with an Archimedean scan is the sampling density. This can be seen simply by recognizing that the number of points scanned in time $t$ will be proportional to $t$, while the area scanned is approximately proportional to the square of the time, as $t^{2}$. Thus, the sampling density and dose at the sample will vary with position in the image. This is also evident from the corrupted regions close to the edge of the reconstructed images due to very sparse sampling in those areas. Also, due to very dense sampling in the center, the beam dose there will be much larger than the average, resulting in 
extra beam damage. Clearly, if uniform sampling distribution is desired, Eq. 4 reveals that we should investigate solutions with $a=0.5$.

\section{Fermat spiral}

Here, we use a different spiral with $a=0.5$ and $b=1$ to give both uniform sampling and constant angular velocity:

$$
x=\sqrt{t} \cos (\omega t), y=\sqrt{t} \sin (\omega t)
$$

This spiral has been known as Fermat spiral, which has the more general form $r^{2}=\omega t$. Since the square root has two solutions (positive and negative), a natural approach is to use one part as the outward scan and the other as the inward scan. Figure 4a shows how $x$ and $y$ change as a function of time for the outward scan part. Figure $4 \mathrm{~b}$ shows the scan path for both outward and inward scans. Note that the end point (A) of the outward scan and the starting point (B) of the inward scan are at opposite sides. Therefore, a smooth wave was added to move the probe from A and B for a smooth transition between outward scan and inward scan. The outward and inward scan paths move clockwise and counter clockwise, respectively. The two reconstructed STEM images are shown in Fig. 4c. Again, the distortion seems to be purely linear due to constant angular velocity. The rotation distortions are opposite as expected from different spiral rotation directions. However, the image quality is not uniform; the edge area is noticeably more blurred than the center area. This non-uniformity is attributed to the anisotropic sample spacing. Near the center area, the spacing between adjacent points along the tangent direction is much shorter than the spacing along radial directions. For the edge area, the spacing along the radial direction is much longer than along the tangential direction. Therefore, despite the nominally uniform areal distribution, the actual sampling is still not ideal.

\section{Constant linear velocity spiral}

We seek a spiral that retains the constant sampling density, but where the distance between samples is isotropic. The solution is known as a constant linear velocity spiral. From the previous discussion on the physical properties of spirals, the two parameters should satisfy $a=0.5$ and $a+b=1$. The spiral equation is thus:

$$
x=\sqrt{t} \cos (\omega \sqrt{t}), y=\sqrt{t} \sin (\omega \sqrt{t})
$$

This spiral has both constant sampling density (dose distribution) and, evenly, isotropic spaced points. A similar scan path was proposed for atomic force microscopy (AFM) [20]. The scan path is shown in Fig. 5a. Examples of the sampling trajectories for both outward and inward

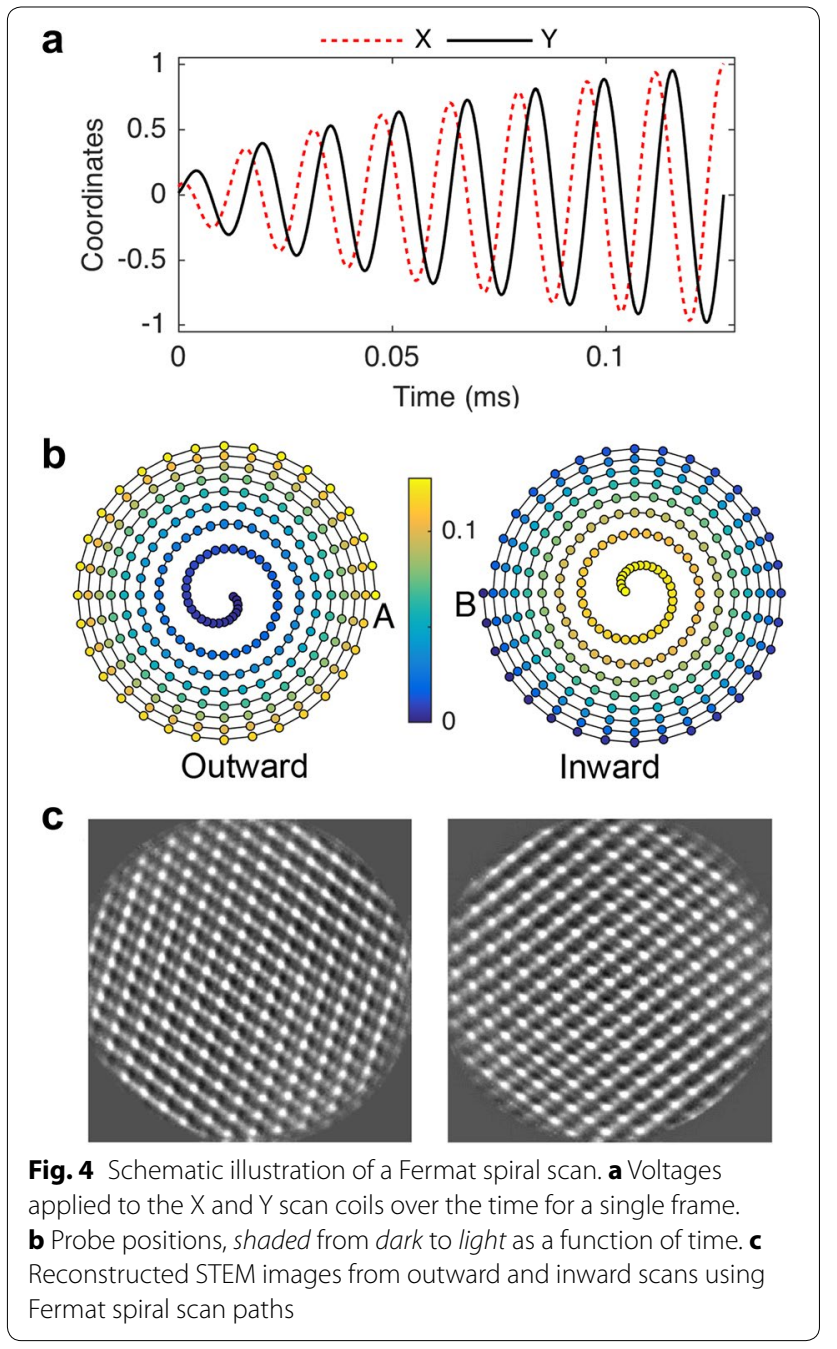

scans are shown in Fig. 5b, where we can see that the data points are evenly distributed along both tangential and radial directions.

Experimental images with the constant velocity spiral are shown in Fig. 5c. The outward and inward parts of the scan are displayed separately. Significant distortions are apparent at the center of the images where the scan frequency is changing the fastest. The two images have opposite rotation distortion directions in the center, which result from different spiral rotation directions. Therefore, the drawback of this spiral is that the angular frequency changes. Since the distortions depend on frequency, the disadvantage is that the distortions are non-uniform across a single frame. Another way to look at this problem is that to keep a constant linear velocity, the angular velocity has to be large near the center and smaller at the edges. Thus, the angular distortion changes with angular velocity, which results in much more severe distortions at the center. 


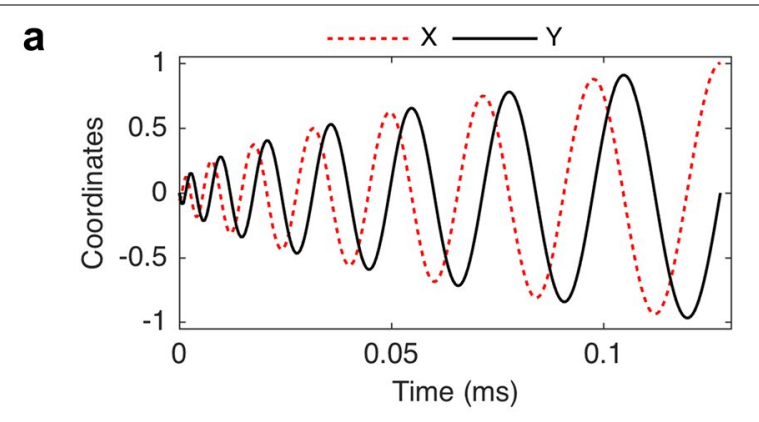

b
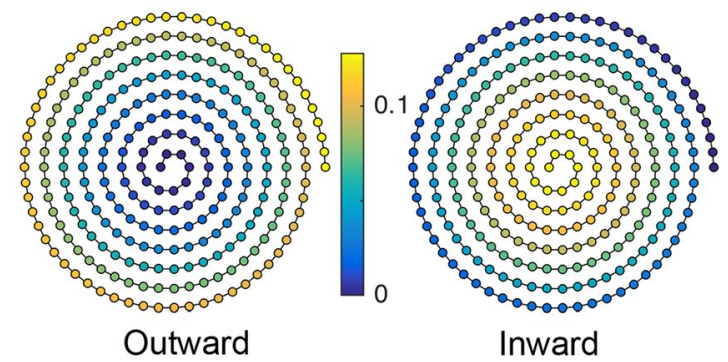

C
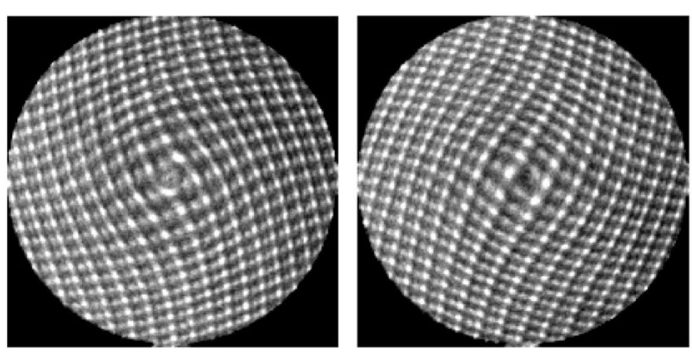

Fig. 5 Schematic illustration of a constant linear velocity scan. a Voltages applied to the $X$ and $Y$ scan coils over the time for a single frame. b Probe positions, shaded from dark to light as a function of time. c Reconstructed STEM images from outward and inward scans using constant linear velocity scan paths

\section{Discussion}

All three spiral scans we tested have successfully eliminated the flyback delay common in conventional STEM. Both Archimedean and Fermat scans yield STEM images with a quality comparable with conventional scan paths, but both have problems with sampling density (dose distribution). The constant linear velocity scan solves the sampling problem but introduces significant distortion in the center. For ease of use, the Fermat scan seems to be the best choice due to its relatively uniform sampling density and easy interpretation of the reconstructed image.

A possible solution to the sampling problem might be truncated spiral functions, which have the same functional form but start from some finite $t_{0}$ instead of from zero. Spirals with varying $a$ and $b$ could also be investigated in future work.

As the drift distortion depends critically on the relative drift direction with respect to the scan direction [2], the varying scan directions in spiral scans lead to an abundance of information for further drift correction within one frame. Other areas for future work could involve hybrid scans, scans adapted on the fly, or changes in the dwell-time per pixel.

\section{Conclusion}

We have demonstrated for the first time that aberration-corrected STEM images can be acquired at high speed with different spiral scans. By completely eliminating the flyback effect in STEM imaging, the spiral scans provide new possibilities to reduce beam damage, image distortion, and drift distortion. Combined with conventional image processing methods, the spiral scans can be used to significantly improve the quality of STEM images. In the future, this system could be extended with high-speed feedback in the FPGA unit. Such capabilities could allow dynamic position correction or atom tracking in hardware, without having to wait for relatively slow data transfers to and from a computer.

\section{Authors' contributions \\ SJ built the scan control system. ARL, RRU, MC, and SJ interfaced the control- ler to the microscope and performed the microscopy experiments. XS and ARL drafted the manuscript. SJ, ARL, RRU, MC, AYB, and SVK conceived and designed the study. XS, SJ, EE, and RKA participated in image analysis. All authors read and approved the final manuscript.}

\section{Author details}

${ }^{1}$ Center for Nanophase Materials Sciences, Oak Ridge National Laboratory, Oak Ridge, TN 37831, USA. ${ }^{2}$ Institute for Functional Imaging of Materials, Oak Ridge National Laboratory, Oak Ridge, TN 37831, USA. ${ }^{3}$ Materials Sciences and Technology, Oak Ridge National Laboratory, Oak Ridge, TN 37831, USA ${ }^{4}$ Computer Science and Mathematics, Oak Ridge National Laboratory, Oak Ridge, TN 37831, USA.

\section{Acknowledgements}

Research supported by Oak Ridge National Laboratory's (ORNL) Center for Nanophase Materials Sciences (CNMS), which is a U.S. Department of Energy (DOE), Office of Science User Facility (XS, RRU, MC, SVK, SJ), by the Division of Materials Sciences and Engineering, Office of Basic Energy Sciences, DOE (ARL and AYB), by ORNL's Laboratory Directed Research and Development Program, which is managed by UT-Battelle LLC for the U.S. DOE (SJ) and by the Office of Advanced Scientific Computing Research, Applied Mathematics program under the ACUMEN project.(EL and RKA).

This manuscript has been authored by UT-Battelle, LLC under Contract No. DE-AC05-000R22725 with the U.S. Department of Energy. The United States Government retains and the publisher, by accepting the article for publication, acknowledges that the United States Government retains a non-exclusive, paid-up, irrevocable, world-wide license to publish or reproduce the published form of this manuscript, or allow others to do so, for United States Government purposes. The Department of Energy will provide public access to these results of federally sponsored research in accordance with the DOE Public Access Plan (http://energy. gov/downloads/doe-public-access-plan).

\section{Competing interests}

The authors declare that they have no competing interests.

Received: 30 March 2016 Accepted: 27 May 2016

Published online: 13 June 2016 


\section{References}

1. Egerton, R.F.: Electron energy-loss spectroscopy in the electron microscope. Springer, Berlin (2011)

2. Sang, X., LeBeau, J.M.: Revolving scanning transmission electron microscopy: correcting sample drift distortion without prior knowledge. Ultramicroscopy 138, 28-35 (2014)

3. Yankovich, A.B., Berkels, B., Dahmen, W., Binev, P., Sanchez, S.I, Bradley, S.A., Li, A., Szlufarska, I., Voyles, P.M.: Picometre-precision analysis of scanning transmission electron microscopy images of platinum nanocatalysts. Nat Commun 5, 4155 (2014)

4. Jones, L., Nellist, P.D.: Identifying and correcting scan noise and drift in the scanning transmission electron microscope. Microsc Microanal 19, 1050-1060 (2013)

5. Ophus, C., Ciston, J., Nelson, C.T.: Correcting nonlinear drift distortion of scanning probe and scanning transmission electron microscopies from image pairs with orthogonal scan directions. Ultramicroscopy 162, 1-9 (2016)

6. Jones, L., Yang, H., Pennycook, T.J., Marshall, M.S.J., Van Aert, S., Browning, N.D., Castell, M.R., Nellist, P.D.: Smart align—a new tool for robust nonrigid registration of scanning microscope data. Adv Struct Chem Imaging $\mathbf{1}, 8(2015)$

7. Lin, J., Cretu, O., Zhou, W., Suenaga, K., Prasai, D., Bolotin, K.I., Cuong, N.T., Otani, M., Okada, S., Lupini, A.R., Idrobo, J.-C., Caudel, D., Burger, A., Ghimire, N.J., Yan, J., Mandrus, D.G., Pennycook, S.J., Pantelides, S.T.: Flexible metallic nanowires with self-adaptive contacts to semiconducting transition-metal dichalcogenide monolayers. Nat Nanotechnol 9 , 436-442 (2014)

8. Ishikawa, R., Mishra, R., Lupini, A.R., Findlay, S.D., Taniguchi, T., Pantelides, S.T., Pennycook, S.J.: Direct observation of dopant atom diffusion in a bulk semiconductor crystal enhanced by a large size mismatch. Phys Rev Lett 113, 155501 (2014)

9. Zan, R., Ramasse, Q.M., Bangert, U., Novoselov, K.S.: Graphene reknits its holes. Nano Lett. 12, 3936-3940 (2012)

10. Kotakoski, J., Mangler, C., Meyer, J.C.: Imaging atomic-level random walk of a point defect in graphene. Nat. Commun. 5, 3991 (2014)
11. de Jonge, N., Peckys, D.B., Kremers, G.J., Piston, D.W.: Electron microscopy of whole cells in liquid with nanometer resolution. Proc Natl Acad Sci USA 106, 2159-2164 (2009)

12. van Heel, M., Gowen, B., Matadeen, R., Orlova, E.V., Finn, R., Pape, T., Cohen, D., Stark, H., Schmidt, R., Schatz, M., Patwardhan, A.: Single-particle electron cryo-microscopy: towards atomic resolution. Q Rev Biophys 33 , 307-369 (2000)

13. Zhou, W., Oxley, M.P., Lupini, A.R., Krivanek, O.L., Pennycook, S.J., Idrobo, J.-C.: Single atom microscopy. Microsc Microanal 18, 1342-1354 (2012)

14. Pennycook, T.J., Lupini, A.R., Yang, H., Murfitt, M.F., Jones, L., Nellist, P.D.: Efficient phase contrast imaging in STEM using a pixelated detector. Part 1: experimental demonstration at atomic resolution. Ultramicroscopy 151, 160-167 (2015)

15. Stevens, A., Yang, H., Carin, L., Arslan, I., Browning, N.D.: The potential for Bayesian compressive sensing to significantly reduce electron dose in high-resolution STEM images. Microscopy. 63, 41-51 (2014)

16. Ziegler, D., Meyer, T.R., Farnham, R., Brune, C., Bertozzi, A.L., Ashby, P.D.: Improved accuracy and speed in scanning probe microscopy by image reconstruction from non-gridded position sensor data. Nanotechnology. 24, $335703(2013)$

17. Ovchinnikov, O.S., Jesse, S., Kalinin, S.V.: Adaptive probe trajectory scanning probe microscopy for multiresolution measurements of interface geometry. Nanotechnology. 20, 255701 (2009)

18. Sanchez, A.M., Galindo, P.L., Kret, S., Falke, M., Beanland, R., Goodhew, P.J.: An approach to the systematic distortion correction in aberrationcorrected HAADF images. J. Microsc. 221, 1-7 (2006)

19. Cook T.A.: Spirals in nature and art: A study of spiral formations based on the manuscripts of Leonardo Da Vinci (1903). Literary Licensing LLC (2014)

20. Mahmood, I.A., Reza Moheimani, S.O.: Spiral-scan atomic force microscopy: a constant linear velocity approach. 2010 10th IEEE Conf. Nanotechnology. NANO 2010, 115-120 (2010)

\section{Submit your manuscript to a SpringerOpen ${ }^{\circ}$ journal and benefit from:}

- Convenient online submission

- Rigorous peer review

- Immediate publication on acceptance

- Open access: articles freely available online

- High visibility within the field

- Retaining the copyright to your article 